\title{
Cognitive functioning after pallidotomy for refractory Parkinson's disease
}

Kenneth Perrine, Michael Dogali, Enrico Fazzini, Djorje Sterio, Edwin Kolodny, David Eidelberg, Orrin Devinsky, Aleksandar Beric

\begin{abstract}
Background-Earlier approaches to pallidotomy for refractory Parkinson's disease had significant complication rates. More recent approaches show fewer complications, but the effect of pallidotomy on cognition is unclear. The current study was conducted to examine the neuropsychological effects of unilateral pallidotomy.

Methods-Neuropsychological testing was performed on patients with medically refractory, predominantly unilateral Parkinson's disease at baseline and after unilateral ventral pallidotomy $(n=28)$ or after an equivalent period without surgery in control patients $(n=10)$.

Results-Pallidotomy patients showed no significant changes from baseline to retesting relative to the control group for any measure. Across all of the tests administered, only five of the surgery patients showed a significant decline, and of these five none declined on more than one test. Depression did not relate to preoperative or postoperative cognition. The pallidotomy group showed a significant improvement in motor functioning and activities of daily living whereas the control group did not. These measures were not associated with the neuropsychological test scores at baseline or retest. Conclusions-Stereotactic unilateral ventral pallidotomy does not seem to produce dramatic cognitive declines in most patients.
\end{abstract}

(F Neurol Neurosurg Psychiatry 1998;65:150-154)

Keywords: Parkinson's disease; pallidotomy; neuropsychological tests

Neurosurgery,

University of

California, Irvine, CA,

USA

M Dogali

Department of Neurology, Cornell University Medical Center and North Shore University Hospital, USA

D Eidelberg

Correspondence to: Dr Kenneth Perrine, Department of Neurology, Hospital for Joint Diseases, 301 East 17 th Street, New York, NY 10003, USA.

Telephone 001212598

6558; fax 0012125986169 .

Received 22 January 1997 and in revised form

5 January 1998

Accepted 13 January 1998 tal functions ${ }^{13-15}$ independent of the primary motor deficit. Patients with left body side signs (and presumably right basal ganglia impairment) may show more impaired cognitive functioning. ${ }^{6}{ }^{16-20}$ Patients with right body side signs may show more impairment for verbal material and those with left body side signs may be more impaired for visuospatial

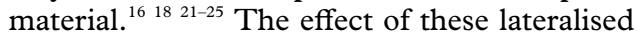
differences and material specificity on postoperative cognitive status is not known.

Cognitive changes after pallidotomy could be expected to occur in some functions based on prior lesion studies of the globus pallidus and the anatomical connections of the basal ganglia with cortical regions involved in cognition. Lesions to the globus pallidus can produce impaired attentional abilities and reductions in planning and organisation. ${ }^{26}$ The globus pallidus has primary efferents to the thalamus and through the thalamus to primary and supplementary motor cortex ${ }^{27}$ and to limbic regions. ${ }^{28}$ Surgical lesions to the globus pallidus could thus impact on the initiation functions mediated by the supplementary motor cortex and the various cognitive functions affected by limbic cortex.

The current study examines cognitive functioning before and after unilateral stereotactic pallidotomy for refractory Parkinson's disease. Patients who have undergone pallidotomy are compared with a comparable group of nonoperated patients with Parkinson's disease.

\section{Methods}

PATIENT SELECTION

Thirty eight patients with refractory Parkinson's disease were recruited after protocol approval by the Institutional Review Boards of the Hospital for Joint Diseases and NYU Medical Center. All eligible patients had disabling fluctuations in motor functioning despite optimal medication management. Inclusion required a diagnosis of parkinsonism with a response to levodopa, the presence of bradykinesia and rigidity, and fluctuations between "off" parkinson symptoms and "on" dyskinesias. Excluded patients had known causes of parkinsonism (for example, encephalitis or neuroleptic treatment), focal brain abnormality on MRI, dementia on mental status testing, supranuclear gaze palsy, ataxia, orthostatic hypotension (blood pressure drop $>30 \mathrm{~mm} \mathrm{Hg}$ on standing), or coexisting illnesses that would complicate assessment or treatment (for example, spinal stenosis, cerebrovascular disease, metabolic disorders).

Twenty eight patients underwent stereotactic ventral pallidotomy (15 men, 13 women; 14 
left side, 14 right side). Ten other patients (eight men, two women) were interested in pallidotomy but did not wish at the time of their initial evaluation to undergo surgery. These patients chose to forego surgery primarily because of lack of knowledge concerning the long term outcome of pallidotomy, and served as unoperated controls (three with left side symptoms, seven with right side symptoms). The surgery group did not differ significantly from the control group for age (combined mean 60.1 (SD 10.0) years), duration of Parkinson's disease (combined mean 13.2 (SD 4.9 years)), levodopa medication dosage at first evaluation (combined mean $825.0 \mathrm{mg}$ ), or side of symptoms.

\section{EVALUATIONS}

All patients underwent at least two comprehensive evaluations. Details of the examinations were presented previously. ${ }^{29}$ The first baseline evaluations were before surgery in the operative group. Subsequent evaluations were between 3 and 12 months later (postoperative for the surgery group, repeated testing without surgery for the control group). Data for each follow up evaluation were not available for all patients. Each evaluation consisted of neurological examination, including videotaped quantification of "on" and "off" states and motor functioning by adaptations of the Core Assessment Program for Intracerebral Transplantation (CAPIT) ${ }^{30}$ and Unified Parkinson's Disease Rating Scale (UPDRS). ${ }^{31}$

Neuropsychological testing was conducted during the "on" state, with no data collected during "off" states, to minimise the deleterious effects of bradykinesia and bradyphrenia. Although dyskinesias were present in many patients during the neuropsychological testing, they were generally not sufficiently severe as to interfere with the tasks administered. Tests requiring a motor response (except for the trail making test) were not included in the battery to minimise the effect of these dyskinesias on the results.

OPERATIVE PROCEDURE

A Leksell stereotactic G-frame was placed and the centre aligned with the midpoint of the anterior commissural-posterior commissural (AC-PC) line. Axial MRI slices at $3 \mathrm{~mm}$ intervals were acquired inferior to the AC-PC line. The MRI images were digitised and transferred to a workstation (Cass/Midco, San Diego, CA, USA). The medial ventral global pallidus target contralateral to the body side with the most prominent symptoms was identified. The target was between 17 to $25 \mathrm{~mm}$ lateral to the midline, 6 to $8 \mathrm{~mm}$ inferior to the AC-PC line, and 2 to $3 \mathrm{~mm}$ anterior to the midcommissural point. A $3 \mathrm{~mm}$ twist drill was made in the same plane as the $x$ coordinate lateral to the midline at an angle of $28^{\circ}$ to $50^{\circ}$ to the AC-PC line. The global pallidus target was verified by intraoperative electrical stimulation, impedance measurements, and single cell recordings. ${ }^{32} \mathrm{~A}$ lesioning electrode was then introduced using stereotactic coordinates from the computerised scan images. The electrode produced a cylin-

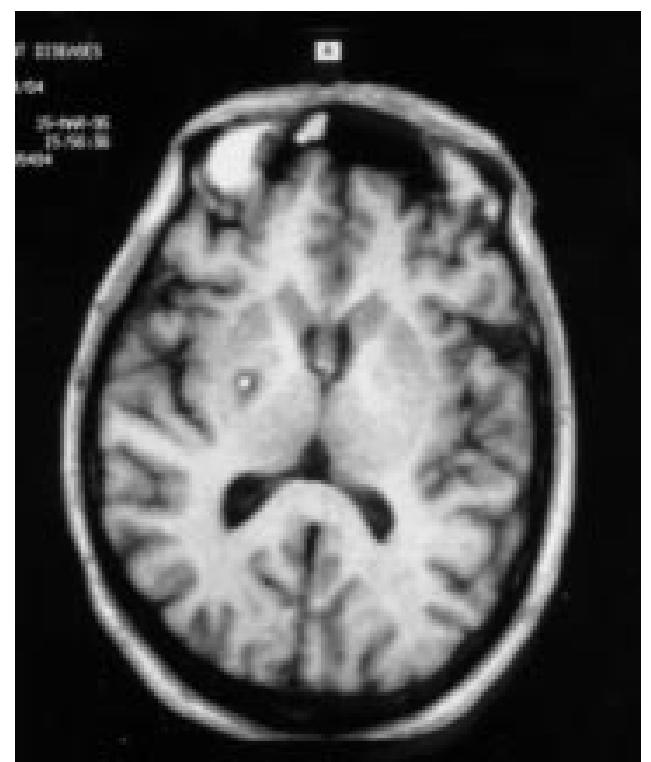

Axial, T1 Weighted MRI showing postoperative lesion in the right globus pallidus.

drical lesion consisting of multiple (three to six) overlapping lesions at $2 \mathrm{~mm}$ intervals by heating the tip to $80^{\circ} \mathrm{C}$ for 60 seconds. Lesion localisation in the global pallidus was confirmed by postoperative MRI (figure).

\section{MEASURES}

The activities of daily living (ADL) and motor scores from the UPDRS were selected as measures of preoperative and postoperative motor functioning. Scores from the neuropsychological battery administered preoperatively were compared with those on follow up testing. Pallidotomy patients had more frequent follow up testing at longer intervals than unoperated controls due to difficulties inherent in recruiting the unoperated group for multiple follow up assessments. Consequently, scores from more recent follow up testing for pallidotomy patients and from later follow up testing for unoperated controls were selected for comparison. However, even with this selection, the unoperated control patients had significantly shorter (mean $=4.5$ (SD 1.6 months)) follow up neuropsychological testing than pallidotomy patients (mean 8.3 (SD 3.6 months; $t=3.25, \mathrm{p}<0.003)$ ). To control for this difference, months between initial and follow up testing was covaried in statistical tests. Five of the 24 pallidotomy patients had neuropsychological testing before the follow up visit used for data in this study.

The neuropsychological battery was based on previous research on Parkinson's disease,$^{3-15}$ with the tests chosen on the basis of their sensitivity to the disease. The battery assessed overall mental status with the mini mental state examination. ${ }^{33}$ Language was assessed by the controlled oral word association test ${ }^{34}$ to quantify verbal fluency. Visual scanning, motor speed, and cognitive flexibility were measured by part A and part B of the trail making test. ${ }^{35}$ Sustained concentration and cognitive processing speed without a motor component were assessed by the oral 


\begin{tabular}{llllll}
\hline & \multicolumn{2}{l}{ Control $(n=10)$} & & & \multirow{2}{*}{ Surgery $(n=28)$} \\
\cline { 2 - 3 } \cline { 5 - 6 } & Baseline & Retest & & Baseline & Retest \\
\hline Mini mental state & $26.7(3.2)$ & $28.4(1.8)$ & & $28.3(1.7)$ & $27.5(1.8)$ \\
Stroop word & $40.0(14.8)$ & $38.0(12.8)$ & & $45.3(10.2)$ & $42.9(12.4)$ \\
Stroop colour & $32.0(9.9)$ & $32.9(16.4)$ & & $37.7(12.8)$ & $36.2(10.6)$ \\
Stroop colour word & $39.1(9.6)$ & $39.3(7.8)$ & & $41.0(9.3)$ & $40.0(11.0)$ \\
Trails A & $64.9(64.5)$ & $59.6(27.6)$ & & $52.2(38.7)$ & $50.4(28.8)$ \\
Trails B & $103.2(55.4)$ & $105.3(24.8)$ & & $140.7(105.0)$ & $128.9(70.5)$ \\
Symbol digit modalities & $35.0(8.2)$ & $42.4(6.2)$ & & $36.5(14.0)$ & $40.6(12.9)$ \\
WCST ${ }^{\star}$ errors & $22.2(12.9)$ & $20.3(14.2)$ & & $26.2(11.7)$ & $22.5(11.9)$ \\
WCST perseverative responses & $13.8(11.7)$ & $10.7(7.8)$ & & $17.6(10.7)$ & $11.5(7.8)$ \\
WCST non-perseverative errors & $10.2(5.9)$ & $10.8(9.5)$ & & $12.0(7.5)$ & $12.3(8.3)$ \\
Hooper & $19.9(4.2)$ & $19.7(6.3)$ & & $19.6(5.5)$ & $24.2(12.1)$ \\
Verbal fluency & $41.6(18.4)$ & $46.5(13.5)$ & & $40.2(13.6)$ & $40.1(14.4)$ \\
Beck depression inventory & $14.8(7.6)$ & $13.1(6.7)$ & & $12.4(7.8)$ & $11.2(6.8)$ \\
California verbal learning test $\dagger$ & NA & NA & & $47.2(12.6) \dagger$ & $46.1(12.2)$
\end{tabular}

*WCST $=$ Wisconsin card sorting test.

†California verbal learning test: sum recalled (subset of $n=17$ ).

version of the symbol digit modalities test $\mathrm{t}^{36}$ and by the Stroop test ${ }^{37}$ scored for the word, colour, and colour-word sections. Concept formation and perseverative tendencies were assessed by the Wisconsin card sorting test ${ }^{38}$ and scored for total errors, perseverative responses, and non-perseverative errors. The Beck depression inventory (BDI) ${ }^{39}$ was administered to quantify self-reported depression. A measure of verbal learning (California verbal learning test $^{40}$ ) was administered to later patients but was unavailable for the preoperative assessment of controls or earlier patients performed at another institution; results are reported for the subset of pallidotomy patients for whom data were available. For the purpose of reporting the percentage of the preoperative sample that was impaired on each test, impairment was defined for an individual patient as a score falling more than $1.5 \mathrm{SD}$ below the mean for that test using normative data from each instrument. This threshold was used based on conventional definitions of psychometric impairment. ${ }^{41}$

DATA ANALYSIS

The primary analysis was a series of one way (surgery/control) analyses of covariance (ANCOVAs) on the postoperative neuropsychological scores using the baseline scores and retest interval in months as covariates. A power analysis from the ANCOVA shows that the effect size exceeds Cohen's "large effect size" criterion of $\mathrm{F}=0.40$ at a power of 0.80 and $\alpha$ of 0.05 using the numbers in the current sample. ${ }^{42}$ The number of patients in the control and surgery samples exceeding a decline of 1.5 SD units (measured at baseline across all patients) at retesting was tallied to examine individual changes, as opposed to the global changes assessed by parametric statistics calculated across individual patients. A paired $t$ test was used to examine changes from baseline to retesting for the ADL and motor scores from the UPDRS. These UPDRS scores were also correlated with neuropsychological test scores at baseline and follow up to determine if change in motor functions was associated with change in cognition.

\section{Results}

There were no significant differences between the 10 controls and 28 pallidotomy patients for any neuropsychological test at baseline or on retesting (table 1). None of the ANCOVA analyses was significant for differences between the pallidotomy and control groups. The baseline score covariate was significant in all analyses.

Examining individual patients, no control patient declined by more than 1.5 SD from baseline to follow up testing for any score. Two pallidotomy patients showed a decline of more than 1.5 SD units from baseline to follow up testing for the mini mental state examination (30 to 26 and 29 to 25 ), but neither had a postoperative score in the demented range. Two patients declined from baseline to follow up on the Stroop word score ( $\mathrm{T}=46$ to 20 and $\mathrm{T}=56$ to 35). One pallidotomy patient declined on the controlled oral word association test ( 55 to 29). No patient declined on more than one test, and there was no clear pattern to the decline seen on single tests.

The total score from the BDI did not differ significantly between pallidotomy and control groups at baseline or follow up evaluations. The depression score did not correlate significantly with any cognitive measure at baseline or follow up. Only one surgery patient declined significantly on the BDI (0 to 13).

The UPDRS scores did not differ significantly at baseline between the pallidotomy group (ADL 17.4 (SD 7.8), motor 33.2 (SD 11.8)) and control group (ADL 17.8 (SD 8.8), motor 27.1 (SD 17.2)). The pallidotomy group improved significantly from baseline to postoperative testing for both the UPDRS ADL score $(t=8.64, \mathrm{p}<0.0001$, postoperative $\mathrm{ADL}=6.6$ (SD 5.9)) and the UPDRS motor score $(t=13.37, \mathrm{p}<0.0001$, postoperative motor score $=10.0$ (SD 6.6)). The control group did not change significantly from baseline to follow up testing for either the ADL score (20.6 (SD $11.6)$ ) or the motor score (31.6 (SD 16.2)). Neither the ADL score nor the motor score correlated significantly with any neuropsychological test at baseline or follow up, and the improvement in ADL and motor scores was 
not associated with change in the neuropsychological test scores.

\section{Discussion}

Pallidotomy did not result in significant deterioration in cognitive functioning. There was no differential change between the control and pallidotomy groups from baseline to retest for any discrete test of cognitive functioning. Only five pallidotomy patients declined by more than 1.5 SD from baseline to postoperative testing, and all five of these patients declined on only one score with no particular pattern to the changes. Depression as assessed by the BDI also showed no significant change between controls and pallidotomy patients, nor any change from baseline to follow up evaluation. There is thus no evidence that pallidotomy produces significant cognitive impairment.

Although some studies report postoperative deficits in language, visuospatial ability, or planning after pallidotomy or thalamotomy, ${ }^{43-48}$ methodological limitations restrict the confidence placed in the these older studies. Some utilised only postoperative data with no preoperative data, ${ }^{44}$ and the postoperative interval was short for most of the studies. ${ }^{43-45}$ Some studies combined pallidotomy and thalamotomy patients, ${ }^{43} 4548$ and none had an adequate description of the operative procedure and specific target to determine the focus of the lesion. Most of the studies reported only global measures of functioning, ${ }^{44} 45$ without inclusion of specific tests of cognitive processing speed, concept formation, and visuospatial ability that are sensitive to the effects of Parkinson's disease. ${ }^{6}$ Some of these older studies examined patients with large bilateral lesions that produced significant morbidity. By contrast, our findings suggest an absence of cognitive morbidity assessed by more discrete tests of cognitive functioning in patients with MRI guided stereotactic unilateral lesions.

Preoperative neuropsychological test scores showed that the patients with Parkinson's disease in this study showed no significant dementia on a screening test, which reflects the exclusionary criteria of global dementia. However, many patients showed mild to moderate impairments in specific areas of cognitive functioning, including cognitive processing speed and visuospatial processing. These findings are consistent with previous neuropsychological findings in Parkinson's disease showing no gross dementia for many patients ${ }^{6218}$ but a preponderance of deficits consistent with frontal $^{911} 1214154950$ and right hemispheric dysfunction. ${ }^{6} 7916$

Many patients also reported mild to moderate depression. Our incidence rate of $38.2 \%$ is comparable with the $46 \%$ rate reported in a review of 14 studies of Parkinson's disease. ${ }^{51}$ However, depression ratings did not correlate significantly with cognitive ability. Other studies also show little relation between depression and degree of motor impairment ${ }^{52}$ or disability. ${ }^{5253}$

Our findings suggest that unilateral pallidotomy in this subset of patients with refractory Parkinson's disease exerts little impact on cognitive functioning. There was no deterioration between groups on any neuropsychological measure. No individual pallidotomy patient declined significantly on more than one test.

Both the pallidotomy and control groups had baseline and repeat testing to compare the groups on test-retest data to control for practice effects. However, the pallidotomy group had a longer mean follow up interval to retesting than the control group. If the lack of significant changes were due to practice effects, then the control group should have improved more than the pallidotomy group due to the shorter test to retest interval. Because the groups did not differ significantly in changes from baseline to retesting, it is unlikely that the findings obtained are due to simple practice effects. The control group was self selected by declining to submit to surgery or postponing surgery, and an argument could be made that this group differed from the group of patients who underwent surgery. However, analyses showed no difference between these two groups for severity of motor symptoms, medication, or cognitive ability. Although some systematic bias between the control and operative groups cannot be completely ruled out, there was no obvious difference in the primary variables of interest in this study.

Unilateral stereotactic ventral pallidotomy thus seems to have little associated postoperative cognitive morbidity. However, our sample was restricted to patients with lateralised motor symptoms and significant fluctuations between "on" dyskinesias and "off" parkinsonism. The effects of pallidotomy in less lateralised patients and the effects of bilateral pallidotomy on cognitive function remain unknown.

1 Marsden CD, Parkes JD. Sources and problems of ong-term levodopa therapy in Parkinson's disease. Lancet $1977 ; \mathrm{i}: 345-9$.

2 Laitinen LV, Bergenheim T, Hariz MI. Leksell's posteroventral pallidotomy in the treatment of Parkinson's disease. $\mathcal{f}$ Neurosurg 1992;76:53-61.

3 Benson DF. Parkinsonian dementia: cortical or subcortical. Adv Neurol 1984;40:235-41.

4 Cummings J, Benson D. Subcortical dementia: Review of an Cummings J, Benson D. Subcortical dementia: Re

5 Girotti F, Carella F, PiaGrassi M, et al. Motor and cognitive performance of parkinsonian patients in the on and off phases of the disease. I Neurol Neurosurg Psychiatry 986;49:657-60

6 Raskin S, Borod J, Wasserstein J, et al. Visuospatial orientation in Parkinson's disease. Int $\mathcal{F}$ Neurosci 1990;51:9-18.

7 Boller F, Passafiume D, Keefe N, et al. Visuospatial impairment in Parkinson's disease: role of perceptual and motor factors. Arch Neurol 1984;41:485-90.

8 DellaSalla S, DiLorenzo G, Giordana A, et al. Directional forecast: a specific visuo-spatial impairment of parkinsonians. F Neurol Neurosurg Psychiatry 1986;49:1258-65.

9 Brown R, Marsden C. Visuospatial function in Parkinson's disease. Brain 1986;109:987-1002.

10 ElAwar M, Becker J, Hammond K, et al. Learning deficit in Parkinson's disease: comparison with Alzheimer's disease Parkinson's disease: comparison with Alzheim

11 Hietanen $M$, Teravainen $\mathrm{H}$. Cognitive performance in early Parkinson's disease. Acta Neurol Scand 1986;73:151-9.

12 Pillon B, Dubois B, Lhermitte F, et al. Heterogeneity of cognitive impairment in progressive supranuclear palsy, Parkinson's disease and Alzheimer's disease. Neurology 1986;36:1179-85.

3 Weingartner $\mathrm{H}$, Burns $\mathrm{S}$, Diebel $\mathrm{R}$, et al. Cognitive impairments in Parkinson's disease: distinguishing between effort-demanding and automatic cognitive processes. Psychiatry Res 1984;11:223-35.

14 Raskin S, Sliwinski M, Borod J. Verbal fluency in Parkinson's disease. F Clin Exp Neuropsychol 1989;11:92.

15 Stern Y, Sano M, Mayeaux R. Comparisons of dementia and intellectual change in Parkinson's and Alzheimer's disease. F Clin Exp Neuropsychol 1987;9:66.

16 Bowen FP. Behavioral alterations in patients with basal ganglia lesions. In: Yahr M, ed. The basal ganglia. New York: Raven Press, 1976:169-80. 
17 Chouza C, Romera S, Laguardia G, et al. Hemiparkinsonism: clinical, neuropsychological, and tomographical studies. In: Hassler R, Christ J, ed. Advances in

18 Seurology. New York: Raven Press, 1984:415-25. logical disturbance in hemiparkinson's disease. Neurology 1987;37:1762-4

19 Tomer R, Levin BE, Weiner WJ. Side of onset of motor symptoms influences cognition in Parkinson's disease. Ann Neurol 1993;34:579-84.

20 Villardita C, Smimi P, Zappala G. Visual neglect in Parkinson's disease. Arch Neurol 1983;40:737-9.

21 Huber S, Shuttleworth E, Freidenberg D. Neuropsychological differences between the dementias of Alzheimer's and Parkinson's disease. Arch Neurol 1989;46:1287-91.

22 Levin BE, Llabre MM, Reisman S, et al. Visuospatial impairment in Parkinson's disease. Neurology 1991;41:3659.

23 Spicer KB, Roberts RJ, LeWitt PA. Neuropsychological performance in lateralized parkinsonism. Arch Neurol performance in

24 Starkstein S, Leiguardia R. Neuropsychological correlates of brain atrophy in Parkinson's disease: a CT-scan study. Mov Disord 1993;8:51-5.

25 Taylor A, Saint-Cyr J, Lang A. Parkinson's disease: cognitive changes in relation to treatment response. Brain 1987;110: $35-51$.

26 Marsden C. The mysterious motor function of the basal ganglia: the Robert Wartenberg lecture. Neurology 1982;32 514-39.

27 Eidelberg D, Moeller R, Ishikawa $\mathrm{T}$, et al. Regional metabolic correlates of surgical outcome following unilateral pallidotomy for Parkinson's disease. Ann Neurol 1996; 39:450-9

28 Parent A, Bellefeuille LD. Organization of efferent projections from the internal segment of globus pallidus in primate as revealed by fluorescence retrograde labeling method. Brain Res 1982;245:201-13.

29 Dogali M, Fazzini E, Kolodny E, et al. Stereotactic ventral pallidotomy for Parkinson's disease. Neurology 1995;45: pallidotomy

30 Langston JW, Widner H, Goetz CG, et al. Core assessment program for intracerebral transplantation (CAPIT). Mov Disord 1992; 7:2-13.

31 Fahn S, Elton RL, Members of the UPDRS Development Committee. Unified Parkinson's disease rating scale. In Fahn S, Marsden CD, Calne DB, et al, eds. Recent developments in Parkinson's disease. Florham Park, NJ: Macmillan Health Care Information, 1987:153-64.

32 Sterio D, Beric A, Dogali M, et al. Neurophysiological properties of pallidal neurons in Parkinson's disease. Ann Neuro 1994;35:586-91.

33 Folstein M, Folstein S, McHugh PJ. Mini-Mental State, a practical method for grading the cognitive state of patients for the clinician. $\mathcal{F}$ Psychiatr Res 1975;12:189-98.
34 Benton AL, Hamsher KS, Varney NR, et al. Contributions to neuropsychological assessment . New York: Oxford University Press, 1983

35 Reitan RM, Wolfson D. The Halstead-Reitan neuropsychological test battery: theory and interpretation. Tucson, AZ: Neuropsychology Press, 1985.

36 Smith A. The symbol digit modalities test: a neuropsychologic test for economic screening of learning and other cerebral disorders. Learning Disorders 1968;3:83-91.

37 Golden CJ. Stroop color and word test . Chicago, IL: Stoelting, 1978.

38 Heaton RK. Wisconsin card sorting test: manual. Odessa, FL: Psychological Assessment Resources, 1981.

39 Beck AT. Beck depression inventory: manual . San Antonio, TX: The Psychological Corporation, 1987.

40 Delis D, Kramer J, Kaplan E, et al. California verbal learning test: adult version. San Antonio, TX: Psychological Corporation, 1987.

41 Lezak M. Neuropsychological assessment. New York: Oxford Press, 1995.

42 Cohen J. Statistical power analysis for the behavioral sciences. 2nd ed. Hillsdale, NJ: 1988:311

43 Darley F, Brown J, Swenson W. Language changes after neurosurgery for parkinsonism. Brain Lang 1975;2:65-9.

44 Delancy Horne D. Performance on delayed response tasks in patients with parkinsonism. F Neurol Neurosurg Psychiatry 1971;34:192-4.

$45 \mathrm{McFie}$ J. Psychological effects of stereotaxic operations for the relief of parkinsonian symptoms. Fournal of Mental Science 1960;106:1512-7.

46 Meier M, Story J. Selective impairment of Porteus maze test performance after right subthalamotomy. Neuropsychologia 1967;5:181-9.

47 Perret K, Kohenof M, Siegfried J. Influences de lesions thalamiques unilaterales sur les fonctions intellectuelles, mnesiques et d'apprentissage de malades parkinsoniens. Neuropsychologia 1969;7:79-88.

48 Riklan M, Levita E. Psychological effects of lateralized basal ganglia lesions: a factorial study. $f$ Nerv Mental Dis 1964;138:233-40.

49 Huber S, Shuttleworth E, Paulson G. Dementia in Parkinson's disease. Arch Neurol 1986;43:987-90.

50 Sagar HJ, Cohen NJ, Sullivan EV, et al. Remote memory function in Alzheimer's disease and Parkinson's disease. Brain 1988;111:185-206.

51 Gotham A, Brown R, Marsden C. Frontal cognitive function in patients with Parkinson's disease on and off levodopa. Brain 1988;111:299-321.

52 Mayeux R, Stern Y, Cote L, et al. Altered serontonin metabolism in depressed patients with Parkinson's disease. Neurology 1984;34:642-6.

53 Horn S. Some psychological factors in Parkinsonism. $f$ Neurol Neurosurg Psychiatry 1974;37:27-31. 